\title{
A Study of Prevalence and Association of Dry Eye Disease with Visual Display Terminal Use in Children- A Cross Sectional Observational Study
}

\author{
Priyanka Sodani ${ }^{1}$, Angli Manhas², Dinesh Gupta ${ }^{3}$, Tabinda Syed ${ }^{4}$, Yang Chen Dolma ${ }^{5}$, Sonika Sangra ${ }^{6}$
}

${ }^{1}$ Department of Ophthalmology, GMC, Kathua, Jammu and Kashmir, India. ${ }^{2}$ Department of Ophthalmology, GMC, Jammu, India. ${ }^{3}$ Department of Ophthalmology, GMC, Jammu, India. ${ }^{4}$ Department of Ophthalmology, GMC, Jammu, India. ${ }^{5}$ Department of Preventive and Social Medicine, GMC, Kathua, Jammu and Kashmir, India. ${ }^{6}$ Department of Preventive and Social Medicine, GMC, Kathua, Jammu and Kashmir, India.

\section{ABSTRACT}

\section{BACKGROUND}

Paediatric dry eye disease is a major health concern. VDT's like laptops, tablets and smart phones are very commonly in use in children. Our study was done to evaluate percentage of DED in children and to evaluate association between VDT use and DED in children.

\section{METHODS}

A cross sectional study was conducted in ophthalmology OPD of a tertiary eye care centre for a duration of one year. All children between 6-16 years of age were included. Exclusion criteria included any ocular surgery in past 6 months or any acute ocular infections, extensive corneal or conjunctival pathology or eyelid pathologies.

\section{RESULTS}

The rate of Dry Eye Disease (DED) found in our study was $11.03 \%$. The daily duration of smartphone use, and total daily duration of VDT use were associated with increased association of dry eye disease. 290 children in all were examined, out of which, 32 had dry eyes. 88 children were excluded according to exclusion criteria. 32 children had Dry Eye Disease and 170 children were taken as non DED group. 97 were males (48.02\%) and 105 were females (51.98\%) out of 202 children included in the study. There were 17 girls $(53.12 \%)$ in the dry eye disease group and 78 girls $(45.88 \%)$ in the non DED group. Smart phone usage per day in DED group was $3.15 \pm 0.97$ hours while in non DED group it was $0.64 \pm 0.69$ hours. Computer usage per day was $1.10 \pm 0.53 \mathrm{hrs}$. in DED group and $0.66 \pm 0.34 \mathrm{hrs}$ in non DED group. Hours used in watching television per day were $1.17 \pm 0.56$ hours in DED group and $1.01 \pm 0.40 \mathrm{hrs}$ in non DED group.

\section{CONCLUSIONS}

There is a strong association between VDT use and dry eye disease in paediatric population. Smart phone usage amongst children should be minimized.

\section{KEY WORDS}

Paediatric, Dry Eye Disease, Smart Phones
Corresponding Author:

Dr. Angli Manhas,

\#381-A, Timber Road,

Indira Colony,

Janipur, Jammu,

Jammu and Kashmir, India.

E-mail: anglimanhas@gmail.com

DOI: $10.14260 / \mathrm{jemds} / 2019 / 802$

Financial or Other Competing Interests: None.

How to Cite This Article:

Sodani P, Manhas A, Gupta D, et al. A study of prevalence and association of dry eye disease with visual display terminal use in children- a cross sectional observational study. J. Evolution Med. Dent. Sci. 2019;8(49):3707-3710, DOI: $10.14260 / \mathrm{jemds} / 2019 / 802$

Submission 04-10-2019,

Peer Review 15-11-2019,

Acceptance 22-11-2019,

Published 09-12-2019. 


\section{BACKGROUND}

Dry eye disease (DED) is a common disorder of eyes affecting a significant percentage of population. Paediatric dry eye disease is a major health concern. Visual display terminals like laptops, electronic tablets, and smart phones are found to be important risk factors for the development of DED; also these are now commonly seen to be used by children.

DED is defined by the report of the definition and classification subcommittee of the International Dry Eye Workshop as a multifactorial disease of the tears and ocular surface, which results in symptoms of discomfort, visual disturbance, and tear film instability, with potential damage to the ocular surface.(1) It is well known multifactorial chronic disorder that is highly prevalent in many countries.(2) It is estimated that number of people affected by dry eye syndrome ranges from 25 to 30 million all over the world.(3) It can occur in association with number of congenital, autoimmune, endocrine, and inflammatory disorders, or under certain environmental and nutritional conditions. (4)

Recent years have seen a dramatic increase in the amount of work performed using visual display terminals (VDTs). VDT use is increasingly common not only in VDT workers but also in the general population due to wide spread use of mobiles and smart phones.(5) The use of VDTs has been accompanied by an increase in symptoms called "video display terminal syndrome", including dry eye disease. It is seen that use of VDTs has been associated with a decreased maximum blink interval and hence the development of dry eye symptoms. In addition many people report ocular fatigue after prolonged work with VDTs. Video display terminal syndrome is not limited to adults as the use of video display terminals has also increased in school children. Availability of number of video games on laptops, large television screens and smart phone has never been as great as it is today and these games have become accessible to almost every child in modern society.(6) Changes in lifestyle and entertainment may be risk factors for dry eye disease in children. Paediatric DED is a public health concern and an economic challenge. Thus it is important to dedicate clinical attention to Paediatric DED.

\section{METHODS}

In this cross sectional study, total 290 children between age group 6-16 years who visited eye OPD of a tertiary eye care centre between Jan. 2018 - Jan. 2019 were included. The study was carried out after obtaining permission from the ethical committee of the institution and was performed according to the tenets of declaration of Helsinki. Those children who fulfilled the criteria of diagnosis of DED were included. Children who underwent any ocular surgery, trauma, acute ocular infection, eyelid problems or with congenital autoimmune disorders were excluded. Children using contact lens were also excluded from the study. Those with past allergic conjunctivitis or with history of any topical steroid medication were excluded. Children excluded from DED with no other ocular complaints other than refraction were taken as non DED group. Due consent was taken from both parents and children. Those suspected of DED from their presenting complaints were subjected to detailed general and ophthalmic history taking. An ophthalmologist performed routine ocular and systemic examination of these children which included visual acuity testing, refraction and evaluation of lid problems, allergic conjunctivitis and keratitis. The children suspected of diagnosis of DED from symptoms, history and routine ocular examination underwent specific diagnostic tests for DED i.e. TBUT (Tear Film Breakup Time), Schirmer's test and slit lamp examination.

\section{Tear Film Breakup Time (TBUT) ${ }^{7}$}

As manipulation of the eyelid or instillation of the anaesthetics can affect the tear film breakup time, the TBUT test was performed before other dry eye tests and recorded after fluorescein staining. Care was taken to avoid contact with the cornea to prevent an excessive reflex secretion of tears.. The time interval between the opening of eyelids and appearance of first dry spot on the tear film was recorded using a stop watch. Three recordings were taken and the average was recorded as the TBUT and considered positive if average tear film breakup time was less than 10 seconds.

\section{Schirmer's Test (Type 1, without Anaesthesia) ${ }^{7}$}

The Schirmer's test was performed after a thorough slit lamp examination so that ocular irritation by the test strip would not interfere with other examination results. The material used was commercially available Whatman no. 41 filter paper strips measuring $35 \times 5 \mathrm{~mm}$ known as Schirmer's tear test filter strips and is folded $5 \mathrm{~mm}$ from one end. The patient was made to sit in a dimly lighted room; the strip folded at the notch was placed gently over the lower palpebral conjunctiva at the junction of lateral $1 / 3$ and medial $2 / 3$. The patient was instructed to keep his eyes open and look straight ahead and blink normally. After 5 minutes, the strips were removed and the amount of wetting in millimetres recorded. The Schirmer's test was considered positive if the length of the wetting was less than $10 \mathrm{~mm}$ at the end of 5 minutes.

Dry Eye Disease was diagnosed using a combination of Questionnaire data and clinical Ophthalmologic testing that gave information on signs and symptoms based on 2007 Dry Eye Workshop (DEWS) guidelines ${ }^{8}$. Positive signs for DED included- TBUT $<10$ seconds, positive corneal and conjunctival surface staining with fluorescein and a modified OSDI score greater than 20 points. Children who showed one or more objective signs and more than 20 points on the modified OSDI score were considered DED positive.

Two Questionnaires- OSDI Questionnaire and Ophthalmologic Questionnaire were used in the study. OSDI questionnaire comprises of questions on ocular irritation symptoms related to dry eye disease and its impact on visual function. This index is subjective parameter of DED diagnosis. Modified OSDI score range from 0-100 points, and higher scores indicate greater eye discomfort due to dry eye disease 9 . Ophthalmologic Questionnaire was designed to obtain information regarding risk factors for dry eye disease, including the mean daily duration of video display terminal (smart phone, television, and computer) use, night time usage 
of smart phones, duration of playing games on smart phone, past history of allergic disease and antihistaminic drug use.

\section{Statistical Analysis}

Data was entered in Microsoft excel and statistical analysis was done using SPSS version 16.0. Results were expressed in the form of numbers and percentages. Association was calculated by Odds ratio. Qualitative data was calculated using Chi Square test and p value less than 0.05 was taken as significant.

\section{RESULTS}

Total 290 children between age group 6-16 years who visited eye OPD of a tertiary eye care centre during the period of one year from Jan 2018 to Jan 2019 were examined by a single ophthalmologist. 88 children were excluded according to exclusion criteria. 32 children had Dry Eye Disease and 170 children were taken as Non-Dry Eye Disease group. 97 males (48.02\%) and 105 females (51.98\%) were there out of 202 children included in the study. There were 17 girls (53.12\%) in the dry eye disease group and 78 girls (45.88\%) in the non DED group. 124 children used smart phones. Rate of smart phone usage was $90.63 \%$ in DED group and $55.88 \%$ in non DED group. Rate of computer use was $65.62 \%$ in DED group and $41.17 \%$ in non DED group whereas rate of TV usage was $62.5 \%$ in DED group and $44.11 \%$ in non DED group. Smart phone usage per day in DED group was $3.15 \pm 0.97$ hours while in non DED group it was $0.64 \pm 0.69$ hours. Computer usage per day was $1.10 \pm 0.53$ hrs. in DED group and $0.66 \pm 0.34$ hrs., in non DED group. Hours used in watching television per day were $1.17 \pm 0.56$ hours in DED group and $1.01 \pm 0.40 \mathrm{hrs}$., in non DED group. Increased usage of smart phones especially more than one hour at night and more than one hour of games led to more symptoms of DED.

The rate of Dry Eye Disease (DED) found in our study was $11.03 \%$. Proportion of DED in smart phone group was 0.90 versus 0.59 in non DED group. Proportion of DED in computer usage group was 0.65 versus 0.41 in non DED group. Proportion of DED in Television group was 0.60 versus 0.51 in non DED group. Odds ratio of DED was found to be 7.1 in smartphone usage group, 2.7 in computer usage group and 10 in television usage group. There is strong association of DED and television and smartphone usage.

\begin{tabular}{|c|c|c|}
\hline Demography & DED Group & Non DED Group \\
\hline Number of subjects & $32(11.03)$ & $170(58.62 \%)$ \\
\hline Mean Age (years) & $10.10 \pm 1.00$ & $11.10 \pm 0.93$ \\
\hline Sex(\% of females) & $17(53.12 \%)$ & $78(45.88 \%)$ \\
\hline \multicolumn{3}{|c|}{ Table 1. Demographic Profile } \\
\hline
\end{tabular}

\begin{tabular}{|c|c|c|c|}
\hline Risk Factor & DED Group & Non DED Group & p Value \\
\hline Use of smart phone (\%) & 90.63 & 55.88 & 0.0003 \\
\hline Use of computer (\%) & 65.62 & 41.17 & 0.01 \\
\hline Use of Television (\%) & 62.50 & 44.11 & 0.03 \\
\hline \multicolumn{4}{|c|}{ Table 2. DED Risk Factors } \\
\hline (p value calculated by Chi Square Test)
\end{tabular}
non DED

\begin{tabular}{|c|c|c|c|}
\hline Risk Factor & DED Group & Non DED Group & OR \\
\hline Smart phone use/day (hrs) & $3.15 \pm 0.97$ & $0.64 \pm 0.69$ & 7.1 \\
\hline Computer use/day (hrs) & $1.10 \pm 0.53$ & $0.66 \pm 0.34$ & 2.7 \\
\hline Television use/day (hrs) & $1.17 \pm 0.56$ & $1.01 \pm 0.40$ & 10 \\
\hline \multicolumn{3}{|l|}{ Table 3. Comparative Analysis of Risk Factors of DED } \\
\hline
\end{tabular}

\section{DISCUSSION}

Our hospital based cross sectional observational study investigated the prevalence of Dry Eye Disease and its association with VDT use especially in paediatric age group as paediatric age group is vulnerable for the use of television and smart phones. Beaver Dam study reported overall prevalence of dry eye disease as $14.4 \%$.(10) Many past studies have reported prevalence of Dry eye disease from 10.8\%$57.1 \%$.11-14) in our study the prevalence of Dry Eye Disease was found to be $11.03 \%$. in a study by Moon J, Kim K, et al the prevalence of DED was $8.3 \%$ in urban group and $2.8 \%$ in rural group.(4)

Various studies have reported ocular adverse effects of smart phones. ${ }^{(4,15)}$ In our study we found a strong association of video display terminal usage especially smart phone and Dry Eye Disease in Paediatric population. This is in agreement with studies from Moon JH et al $(1,4)$, and Wagner RS et al.(16) Rate and mean time spent using VDTs especially smart phones were greater in DED group than in controls. Increased use of smart phones for long hours can affect the tear film and ocular surface, which can lead to multiple ocular symptoms. Blue light emitted from smart phones can damage corneal epithelium.(17) Excessive evaporation of the tear fluid due to prolonged blinking intervals and decreased blink rate while playing games and watching videos on smart phones is considered a causative factor in VDT associated dry eye disease. ${ }^{(18,19)}$ In our study we also found that increased use of smart phones especially at night and more than one hour use of smart phones for games leads to increased occurrence of Dry eye symptoms which needs to be supported by a further study of larger population.

\section{CONCLUSIONS}

The prevalence of Dry Eye Disease in children (6-16 yrs.) is $11.03 \%$. There is a strong association of video display terminal use and Dry Eye Disease in paediatric age group. There should be close observation and cautious use of VDTs especially smart phones amongst paediatric population. With digitalisation the brunt should not be on the eyes of our future generation.

\section{REFERENCES}

[1] Moon JH, Lee MY, Moon NJ. Association between video display terminal use and dry eye disease in school children. J Pediatr Ophthalmol Strabismus 2014;51(2):87-92.

[2] Kawashima M, Uchino M, Yokoi N, et al. Association between dry eye disease and physical activity as well as sedentary behaviour: results from Osaka study. Article ID 943786, Journal of Ophthalmology 2014;2014:6.

[3] Phadatare SP, Momin M, Nighojkar P, et al. A comprehensive review on dry eye disease: diagnosis, medical management, recent developments and future challenges. Article Id 704946, Advances in Pharmaceutics 2015;2015:12. 
[4] Moon JH, Kim KW, Moon NJ. Smartphone use is a risk factor for pediatric dry eye disease according to region and age: a case control study. BMC Ophthalmology 2016;16(1):188.

[5] Uchino M, Yokoi N, Uchino Y, et al. Prevalence of dry eye disease and its risk factors in visual display terminal users: the Osaka study. Am J Ophthalmol 2013;156(4):759-66.

[6] Rechichi C, De Mojà G, Aragona P. Video game vision syndrome: a new clinical picture in children? J Pediatr Ophthalmol Strabismus 2017;54(6):346-55.

[7] Roka N, Shrestha SP, Joshi ND. Association of tear secretion and tear film instability in cases with pterygium and normal subjects. Nepal J Ophthalmol 2013;5(1):16-23.

[8] The Definition and classification of dry eye disease: report of the Definition and Classification Subcommittee of the International Dry Eye Workshop (2007). Ocul Surf 2007;5(2):75-92.

[9] Denoyer A, Rabut G, Bardouin C. Tear film aberration dynamics and vision related quality of life in patients with dry eye disease. Ophthalmology 2012;119(9):18118.

[10] Moss SE, Klein R, Klein BE. Prevalence of and risk factors for dry eye syndrome. Arch Ophthalmol 2000;118(9):1264-8.

[11] Farrell J, Grierson DJ, Patel S, et al. A classification for dry eyes following comparison of tear thinning time with schirmer tear test. Acta Ophthalmol (Copenh) 1992;70(3):357-60.
[12] Toda I, Fujishima H, Tsubota K. Ocular fatigue is the major symptom of dry eye. Acta Ophthalmol (Copenh) 1993;71(3):347-52.

[13] Albietz JM. Prevalence of dry eye subtypes in clinical optometry practice. Optometry Vis Sci 2000;77(7):35763.

[14] Versura P, Cellini M, Torreggiani A, et al. Dryness symptoms, diagnostic protocol and therapeutic management: a report on 1,200 patients. Ophthalmol Res 2001;33(4):221-7.

[15] Kim J, Hwang Y, Kang S, et al. Association between exposure to smart phones and ocular health in adolescents. Ophthalmic Epidemiol 2016;23(4):269-76.

[16] Wagenr RS. Smart phones, video display terminals and dry eye disease in children. J Pediatr Ophthalmol Strabismus 2014;51(2):76.

[17] Lee JB, Kim SH, Lee SC, et al. Blue light induced oxidative stress in human corneal epithelial cells: protective effects of ethanol extracts of various medicinal plant mixtures. Invest Ophthalmol Vis Sci 2014;55(7):4119-27.

[18] Argiles M, Cardona G, Perez-Cabre E, et al. Blink rate and incomplete blinks in six different controlled hard copy and electronic reading conditions. Invest Ophthalmol Vis Sci 2015;56(11):6679-85.

[19] Cardona G, Garcia C, Seres C, et al. Blink rate, blink amplitude and tear film integrity during dynamic visual display terminal tasks. Curr Eye Res 2011;36(3):190-7. 